\title{
Pathogenesis of HIV Malignancy
}

National Cancer Institute

\section{Source}

National Cancer Institute. Pathogenesis of HIV Malignancy. NCI Thesaurus. Code C18923.

Induction or development of a malignant disease(s) or condition(s) that results from retroviral activity or arises due to the immunocompromised state of an HIV infected individual. $(\mathrm{NCl})$ 